\title{
D-Dimer: A Literature Review
}

\author{
Petar Trifonov
}

Clinic of Gastroenterology, UMHAT "St. Ivan Rilski", Sofia, Bulgaria

\begin{abstract}
The liver is responsible for the synthesis and clearance of most of the proteins involved in the two cascadescoagulation and fibrinolysis. Some patients with liver cirrhosis can experience severe bleeding whilst other may have thrombotic complication.

Evaluating the coagulation activity in ascitic fluid, there were solid evidences, that ascitic fluid is a procoagulant. $P T$ and INR in serum increased significantly from Child $A$ to $C$, while fibrinogen decreased. Serum D-Dimer levels were founded higher in cirrhotics with ascites, especially in Child C group, in HCC, in SBP condition and decrease after resolution of ascitic fluid. D-Dimer levels can be lower in serum than in ascitic fluid.

In oncology there are solid evidences for the prognostic value of D-Dimer. Serum D-Dimer is qualified there as a prognostic factor associated with increased mortality risk and shorter overall survival.

More in-depth research is needed towards which levels of D-Dimer in serum or ascites could be a prognostic factor for SBP, for an increased likelihood of portal thrombosis or bleeding from GIT. It can be speculated, that D-Dimer levels can be used as prognostic factor for various complications in liver diseases. The study of D-dimer in hepatology could influence future therapeutic regimens.
\end{abstract}

\section{Keywords: D-Dimer, Ascites, Cirrhosis, SBP}

\section{Introduction}

Coagulation is now recognised as a complicated harmonic process. It includes interactions and balance between procoagulation, anticoagulation, and the fibrinolytic system. The liver is responsible for the synthesis and clearance of most of the proteins involved in the two cascades- coagulation and fibrinolysis. Therefore it plays an important role in the hemostasis.

Researchers assumed that patients with cirrhosis have reduced levels of most coagulation factors and their respective inhibitors, as well as reduced fibrinolytic enzymes. Some of them can experience severe bleeding whilst other may have thrombotic complication. Often there are situations, when these two occure simultaneously. The coagulopathy due to liver cirrhosis is still a challenge for the physicians. The parameters, that can predict such incidents, are difficult to evaluate. That is why the efforts of researchers in recent years have been aimed there. One of the most encouraging tests is D-Dimer. 
D-Dimer molecule was first discovered in 1973 and with it's molecular weight of $180 \mathrm{kDA}$ it is the smallest product of fibrin degradation. [1] Many physicians made the suggestion, that according the levels, we can predict the severity of the cirrhotic complications, hyperfibrinolysis and likelihood of occurrence of spontaneous bacterial peritonitis.

\section{Review Results}

This literature review can be divided in five subsections according to the results in cirrhotic patients, obtained in the different studies.

\section{A. Ascitic fluid as coagulation factor}

Evaluating the coagulation activity in ascitic fluid Thaler et al. added ascitic fluid to platelet-free normal pooled plasma. [2]

- Ascitic fluid was clearly procoagulant as it substantially shortened clotting times of plateletfree normal pooled plasma.

Another experiment investigated coagulation factor $(F)$ activities in ascitic fluid and plasma:

- Extracellular vesicle-associated tissue factor activity ${ }^{1}$ was highly elevated in ascitic fluid compared with plasma of the same patients.

- $\quad$ Coagulation factor FII and FVII activities were low in ascitic fluid.

- $\quad$ FV, FVIII, and FX activities were not detectable in ascitic fluid.

They added also washed ascitic fluid-derived extracellular vesicles to vesicle-depleted normal pooled plasma, which:

- $\quad$ shortened the clotting time.

- Thrombin-antithrombin (TAT) complex, prothrombin fragment 1+2, plasmin-a2-antiplasmin (PAP) complex, and D-dimer levels were highly elevated in ascitic fluid compared with plasma.

- $\quad$ Fibrinogen was not detectable in ascitic fluid. We can speculate that the high level of D- Dimer is related to the conversion of fibrinogen into fibrin in ascitic fluid.

\section{B. D-Dimer and the coagulation factors}

One of the first studies about hyperfibrinolysis in different stages of liver disease dates from 1996, when Francesco Violi et al. divided 67 patients with liver cirrhosis by Child- Pugh classification score. [3] Tissue plasminogen activator antigen and activity, plasminogen activator inhibitor antigen and activity, fibrin/fibrinogen degradation products, and D-Dimer were measured in each patient.

- $\quad$ Patients with normal levels of fibrin/fibrinogen degradation products and high D-Dimer levels showed significant progressive decreases of plasminogen activator inhibitor antigen levels and activity from class $\mathbf{A}$ to class $\mathbf{C}$. There was negative correlation between this decrease and prothrombin time.

1. Tissue factor (TF) is a transmembrane glycoprotein and the main initiator of the blood coagulation cascade. Active TF is absent from the circulation under physiologic conditions. It is the receptor for factor VIIVIIa and is essential for hemostasis. Tissue factor-positive extracellular vesicle (EVTF) are highly procoagulant and can be used as a biomarker of pathologic intravascular TF expression. [10]

- Tissue plasminogen activator levels were not different in the three Child classes. 
- Twenty-five patients (7 in class B and 18 in class $\mathrm{C}$ ) with high circulating levels of fibrin/fibrinogen degradation products and high D-Dimer had higher levels of tissue plasminogen activator and activity and lower levels of plasminogen activator inhibitor antigen and activity. Their suggestion was, that the plasminogen activator inhibitor levels (and therefore hyperfibrinolysis) is strongly related to liver function.

- The inequality between tPA and plasminogen activator inhibitor in patients with high circulating levels of D-Dimer indicates that hyperfibrinolysis may be due mainly to clotting activation.

These results were expanded by Romanelli et al. [4] They evaluated 33 patients, divided by Child- Pugh classification score, and 21 controls. They test Clot Lysis Time (CLT), D-Dimer, tissue plasminogen activator (t-PA), plasminogen activator inhibitor (PAl-1), alpha2-antiplasmin (a2AP), plasminogen (PLG), thrombin activatable fibrinolysis inhibitor (TAFIs). They compared coagulation factor levels between cirrhotic patients and healthy subjects.

The results showed:

- D-Dimer, PAI-1 and t-PA levels were significantly higher and CLT shorter in serum from cirrhotics than from healthy subjects.

- $\quad$ A2AP, PLG, TAFI and fibrinogen levels were lower in serum from cirrhotics than controls.

In 2013 Dhanunjaya $Y$ et al. [5] divided 99 patients by Child- Pugh classification score. From A to C the correlations were:

- $\quad$ The serum D-Dimer levels were found to be increased significantly

- $\quad$ Prothrombin time and INR increased significantly

- $\quad$ Fibrinogen decreased

\section{D- Dimer: Ascitic and serum levels}

In 2008 Spadaro et al. included 70 patients with liver disease in their study evaluating the relation between D- Dimer and the severity of the liver disease. [6] The etiology of the disease was HCV- HBV-infection, past alcohol abuse, autoimmune and combinations. 14 patients were with HCC. Patients were divided into 2 groups: A- presence of ascites and B- without. D-Dimer levels were measured also after disappearance of abdominal fluid.

- Serum D-Dimer mean levels were higher in group A (649 $\pm 420 \mathrm{~g} / \mathrm{L})$ than in group B (359 \pm $219 \mathrm{~g} / \mathrm{L})$.

- After the resolution of ascitic fluid mean D-Dimer levels decreased in all 34 patients with high basal levels, returning to normal range in half of them. In these patients, D-Dimer levels after resolution of ascites (438 $\pm 279 \mathrm{~g} / \mathrm{L}$ ) were not significantly different from those found in patients without ascites (group B).

- They found also, that patients without ascites, but with HCC have higher levels of serum DDimer.

\section{Exchange of D- Dimer between ascitic fluid and serum}

Then the researchers' gaze shifts detecting the D- Dimer levels in ascitic fluid and its role and place in the hyperfibrinolysis. The results from Romanelli et al. `s research showed: [4]

- A2AP, PLG, TAFI and fibrinogen levels were significantly higher in plasma than ascites. 
- D-Dimer levels were significantly lower in serum than in ascitic fluid.

- Similar concentrations in plasma and ascites were found for t-PA and PAI-1.

Taking these data into account, they suggest, that ascitic fluid compartment is in continuous exchange with plasmatic compartment, possibly through lymphatic flux, and contributes to the coagulopathy of liver cirrhosis.

\section{E. D- Dimer as prognostic factor}

In 2014 Weiyi X et al. made a research about D- Dimer as prognostic factor of spontaneuous peritonitis in cirrhotic patients [7]. They observed, that serum D- Dimer and CRP are significantly higher in the group of people with liver cirrhosis and SBP than in the control group. Their work has been significantly expanded in the study of Gohary et al. [8]. The study was conducted among 60 patients with liver cirrhosis classified into four groups: Group I- 15 cirrhotic patients without ascites. Group II- 15 cirrhotic patients with ascites. Group III- 15 cirrhotic patients with ascites and SBP. Group IV - 15 healthy subjects.

Table 1: Mean D- Dimer level in serum and ascitic fluid in different groups

\begin{tabular}{|l|l|l|}
\hline $\mathrm{n}=60$ & $\begin{array}{l}\text { Serum D- Dimer mean } \\
\text { level [mg/L] }\end{array}$ & $\begin{array}{l}\text { Ascitic D- Dimer mean } \\
\text { level [mg/L] }\end{array}$ \\
\hline $\begin{array}{l}\text { Group I } \\
\mathrm{n}=15\end{array}$ & $249.42 \pm 79.01$ & \\
\hline $\begin{array}{l}\text { Group II } \\
\mathrm{n}=15\end{array}$ & $288.24 \pm 215.63$ & $311.69 \pm 137.61$ \\
\hline $\begin{array}{l}\text { Group III } \\
\mathrm{n}=15\end{array}$ & $553.98 \pm 328.02$ & $422.77 \pm 193.40$ \\
\hline $\begin{array}{l}\text { Group IV } \\
\mathrm{n}=15\end{array}$ & $285.38 \pm 64.6$ & \\
\hline
\end{tabular}

- Mean serum D-Dimer was the highest among cirrhotic patients with ascites and SBP compared with mean levels observed among cirrhotic patients without ascites, cirrhotic patients with ascites, and the control group. (Table 1.)

- $\quad 60 \%$ of ascitic fluid D-Dimer levels were higher than serum levels in the same group.

- A significant positive correlation was detected between hemoglobin as well as platelets and serum D-Dimer in group III.

- $\quad$ Significant negative correlation was discovered between serum D-Dimer and splenic size and portal vein diameter.

The current study confirmed that serum D-Dimer among patients with SBP is significantly higher than that in normal and liver diseased participants whilst the higher ascitic fluid level of D-Dimer among SBP patients did not significantly differ from other patients. Moreover, serum D-Dimer showed a valuable diagnostic test for detecting patients with SBP and also for detecting the severity of liver disease. Similar data were obtained in the study of Thaler et al., where in 317 patients the research team observed that serum D-Dimer levels showed a stepwise increase with ascites severity. [2]

Because Spadaro et al. found a higher level of D- Dimer in patients with HCC, we comment additionally the D- Dimer level in patients with other types of cancer. In 2012 Ay, C. et al. assessed the prognostic value of D-dimer levels for overall survival and mortality risk in 1178 cancer patients: [9] 
- $\quad$ An elevated serum D-Dimer level qualified as a prognostic factor associated with increased mortality risk and shorter overall survival in patients with brain tumors, lymphomas and in those with breast, lung, stomach, colorectal, pancreatic and prostate cancers.

\section{Conclusion}

There are solid evidences that D-Dimer can be tested as a marker for advanced liver disease as well as a marker for orccurence of SBP. The mechanisms of the influence of acites on hyperfibrinolysis are insufficiently understood and studied. Therefore two types of research are required: clinical and laboratory. More in-depth research is needed towards which levels of D-Dimer in serum or ascites could be a prognostic factor for SBP, for an increased likelihood of thrombosis of v. portae or bleeding from GIT. The study of Ddimer in hepatology could influence future therapeutic regimens.

\section{References}

1. J. Gaffney. D-dimer. History of the discovery, characterisation and utility of this and other fibrin fragments.Fibrinolysis, Volume 7, Supplement 2, 1993, Pages 2-8

2. Thaler J, Lisman T, Quehenberger P, Hell L, Schwabl P, Scheiner B, Bucsics T, Nieuwland R, Ay C, Trauner M, Pabinger I, Reiberger T, Mandorfer M. Intraperitoneal Activation of Coagulation and Fibrinolysis in Patients with Cirrhosis and Ascites. Thromb Haemost. 2021 May 21.

3. Francesco Violi M.D., Domenico Ferro, Claudio Quintarelli, Antonio Musca, Francesco Balsano, Corrado Cordova, Stefania Basili, The Calc Group. Hyperfibrinolysis Resulting from Clotting Activation in Patients with Different Degrees of Cirrhosis. HEPATOLOGY 1993;17:78-83.

4. Romanelli, R. G., Cellai, A. P., Lami, D., Natucci, F., Tosti-Guerra, C., Abbate, R., ... Laffi, G. (2015). D-dimer and fibrinolytic activity in patients with decompensated liver cirrhosis. Digestive and Liver Disease, 47, e33.

5. Dhanunjaya Y, Usha Anand, and Anand CV. A Study of Plasma D-Dimer Levels in Various Stages of Liver Disease. Dhanunjaya et al., J Liver 2013, 2:2

6. Spadaro A, Tortorella V, Morace C, Fortiguerra A, Composto P, Bonfiglio C, Alibrandi A, Luigiano C, De Caro G, Ajello A, Ferrau O, Freni MA. High circulating D-dimers are associated with ascites and hepatocellular carcinoma in liver cirrhosis. World J Gastroenterol. 2008 Mar 14;14(10):1549-52.

7. Weiyi X, Baode C, Qing X. The application of plasma D-dimer in the diagnosis of spontaneous peritonitis in cirrhotic patients with ascites. Int J Lab Med 2014; 21: 2883-2884

8. El Gohary AM, Elyamany AS, Mikhael NL, Mahmoud MG, Tawfik MMR. Serum and ascitic D-dimer in cirrhotic patients with spontaneous bacterial peritonitis. Clin Exp Hepatol. 2021;7(2):134-140

9. Ay, C., Dunkler, D., Pirker, R., Thaler, J., Quehenberger, P., Wagner, O., ... Pabinger, I. High D-dimer levels are associated with poor prognosis in cancer patients. Haematologica, 97(8), 1158-1164.

10. Clare M Schmedes, Steven P Grover, Yohei M Hisada, Marco Goeijenbier, Johan Hultdin, Sofie Nilsson, Therese Thunberg, Clas Ahlm, Nigel Mackman, Anne-Marie Fors Connolly, Circulating Extracellular Vesicle Tissue Factor Activity During Orthohantavirus Infection Is Associated With Intravascular Coagulation, The Journal of Infectious Diseases, Volume 222, Issue 8, 15 October 2020, Pages 1392-1399

\section{Corresponding author:}

Petar Trifonov, Clinic of Gastroenterology,UMHAT St. Ivan Rilski, Sofia, Bulgaria

Tel: +359888605566 